\title{
Overexpressing the WRKY transcription factor OsWRKY97 improves drought tolerance in rice
}

\section{Miaomiao Iv}

Sichuan Agricultural University

Dejia Hou

Huazhong Agriculture University

Taozhi Ye

Sichuan Agricultural University

Lin Zhang

Sichuan Agricultural University

Jiangbo Fan

Sichuan Agricultural University

Chunliu Li

Sichuan Agricultural University

Yilun Dong

Sichuan Agricultural University

\section{Wenqian Chen}

Sichuan Agricultural University

\section{Songhao Rong}

Sichuan Agricultural University

\section{Yihao Sun}

Sichuan Agricultural University

\section{Jinghong $\mathbf{X u}$}

Sichuan Academy of Agricultural Science

\section{Liangjun Cai}

Sichuan Academy of Agricultural Science

\section{Xiaoling Gao}

Sichuan Agricultural University

Jianqing Zhu

Sichuan Agricultural University

\section{Zhengjian Huang}

Sichuan Agricultural University

Zhengjun Xu ( $\square$ mywildrice@aliyun.com )

Sichuan Agricultural University

Lihua Li ( $\sim$ lilihua1976@tom.com ) 
Sichuan agricultural University

\section{Research article}

Keywords: Drought tolerance, ABA, OsWRKY97, antioxidation, Oryza sativa L.

Posted Date: March 16th, 2020

DOI: https://doi.org/10.21203/rs.3.rs-17373/v1

License: (c) (1) This work is licensed under a Creative Commons Attribution 4.0 International License. Read Full License 


\section{Abstract}

Background: WRKY transcription factor (TF) is one of the largest TF families in plants and plays an important role in plant development and stress protection. However, the function of individual WRKY gene in plants are still under investigation. Here, we identified a new member of WRKY TF family, OsWRKY97, and analyze its role in stress resistance by using a series of transgenic plant lines.

Results: OsWRKY97, which positively regulates drought tolerance in rice. OsWRKY97 was expressed in all examined tissues and could be induced by various abiotic stresses. OsWRKY97 is located in the nucleus. Compared with the wild-type (WT), OsWRKY97 -overexpressing (OE) plants enhanced the tolerance of rice to drought and osmotic stress. In addition, OE plants also showed higher sensitivity to exogenous abscisic acid (ABA). When exposed to drought stress, up-regulation OsWRKY97 increased the accumulation of $\mathrm{ABA}$ and reduced water loss. Furthermore, OE plants achieved higher proline content and reduced levels of malondialdehyde (MDA) and reactive oxygen species (ROS). The homeostasis of ROS was maintained via the activity of active oxygen scavenging enzyme, superoxide dismutase (SOD), catalase (CAT) and peroxidase (POD), activated by OsWRKY97

Conclusions: These results indicate that OsWRKY97 plays a crucial role in the response to drought stress and may possess high potential value in improving drought tolerance in rice.

\section{Background}

Drought is one of the major stresses that seriously affects plant growth and reduces yield[1]. Plants have evolved various strategies to cope with drought stress for survival and development. The response process of plants to drought stress includes stress signal perception, signal transduction and amplification, and adaptation at morphological, physiological and molecular levels[2]. In these processes, many diverse stress-related proteins are expressed that enhance drought resistance via outputs such as osmotic adjustment, stomatal closure, and ROS scavenging[3].

ABA has been characterized extensively an important plant hormone, which responds to drought stress by regulating stomatal closure and transpiration rate $[4,5]$. It has been reported that most water stress inducing genes respond to treatment with exogenous $A B A$ and relate to ABA signal transduction pathways[6]. ABA-mediated stomatal closure is usually accompanied by the production of $\mathrm{H} 2 \mathrm{O} 2[7]$. It was reported that $\mathrm{H} 2 \mathrm{O} 2$ participated in ABA signal transduction in plant guard cells and triggered stomatal closure by activating $\mathrm{Ca} 2+$ channels on cell membranes $[8,9]$. It has also been reported that $\mathrm{H} 2 \mathrm{O} 2$ can induce stomatal closure, which was independent of ABA pathway[10]. However, under stress conditions, the content of $\mathrm{H} 2 \mathrm{O} 2$ increased sharply in plant cells, which posed a threat to plants, but it is also thought that ROS is a signal for activation of defense pathways[11].

The WRKY TF is one of the largest TF families and is a class of DNA-binding proteins in plants[12]. The WRKY protein has a highly conserved WRKY domain, containing an almost unchanged WRKYGQK sequence at the $\mathrm{N}$-terminal, followed by a Cx4 - 5Cx22-23 HxH or Cx7Cx23 HxC zinc-finger motif[13]. 
Based on the number of WRKY domains and the types of zinc finger motifs, WRKY proteins can be divided into groups I-III. Group I WRKY contains two WRKY domains, Group II WRKY contains a WRKY domain, and the I-II groups all contain a $\mathrm{Cx} 4-5 \mathrm{Cx} 22-23 \mathrm{HxH}$ zinc-finger motif. Group III WRKY contains a WRKY domain and a Cx7Cx23 HxC zinc-finger motif[14].

At present, studies have revealed the involvement of the WRKY family in plant responses to abiotic stresses. For example, Overexpression of TaWRKY2 reduced the water loss rate of transgenic wheat and enhanced its drought resistance[15]. Furthermore, many TFs have been reported to be involved in ABAmediated signaling pathways of plant responses to drought stress[16]. CmWRKY10 is located in the nucleus, and overexpression of CmWRKY10 enhanced chrysanthemum tolerance to drought stress through the ABA-signaling pathway[17].OsWRKY45 plays an important role in drought tolerance and ABA signal-regulation in rice[18]. In recent years, progress has been made in ABA sensing signal transduction pathway[19]. In the presence of ABA, the receptor Pyrabactin resistance (PYR) / PYR1-like (PYL) / Regulatory component of ABA receptors (RCARs) of ABA can disrupt the interaction between the SNF1related protein kinase 2 (SnRK2s) and 2C-type protein phosphatases (PP2Cs), thus preventing the PP2Cmediated dephosphorylation of the SnRK2s and resulting in the activation of the SnRK2 kinases[20]. Further analyses revealed that the activation of SnRK2s plays a key role in the ABA-dependent phosphorylation of ABRE-binding protein (AREB)[21]. It has been reported that WRKY TFs regulate the expression of many genes related to ABA-induced physiology and development by binding the w-box sequence in the promoters of AREBs[22]. AtWRKY63 mutants impaired the sensitivity of ABA mediated stomatal closure and affects its drought tolerance[23]. It is reported that WRKY40 and WRKY60 transcription factors inhibit ABI4 gene expression. In addition, their mutual antagonism not only occurs between WRKY40 and WRKY60, but also between WRKY18 and WRKY40 to balance the repressive functions on the ABI4 gene. However, for ABI5 gene, WRKY60, WRKY18 and WRKY40 inhibited ABI5 independently, but WRKY 60 also antagonized the inhibition of WRKY18 and WRKY18-WRKY40 heterodimer to balance the inhibition of ABI5 gene. These findings help to understand the complex mechanism of WRKY mediated ABA signal transduction[24].

The corresponding sequence of OsWRKY97 (LOC_Os12g02420.1) can be downloaded from Rice Genome Annotation Project (RGAP) (http://rice.plantbiology.msu.edu/) and OsWRKY97 belongs to the WRKY family according to the SMART database (http://smart.embl-heidelberg.de/). The OsWRKY97 gene was identified by analyzing rice drought stress microarray data generated by our lab (unpublished). The gene was strongly up-regulated under drought conditions in microarray data. So we think that OsWRKY97 may also play an important role in the response to abiotic stresses. In this study, we found that the expression level of OsWRKY97 in Nipponbare was significantly improved under abiotic stress, and overexpression of OsWRKY97 improved the tolerance of transgenic rice to drought and osmotic stress. OE plants also showed higher sensitivity to exogenous $A B A$ and increased the accumulation of $A B A$ under drought stress. In addition, we found that OE plants had higher activity of active oxygen scavenging enzymes, less membrane lipid peroxidation and higher accumulation of proline under drought conditions. These results suggest that OsWRKY97 positively regulates drought stress tolerance in rice. 


\section{Results}

\section{Expression patterns of OsWRKY97}

We examined the expression of OsWRKY97 in 15 different tissues by quantitative real-time PCR (qRTPCR), so that the expression pattern of OsWRKY97 in different tissues at different stages of rice was detected. OsWRKY97 was expressed in various tissues of rice at different stages (Additional file 1:Figure S1). We investigated the expression patterns of OsWRKY97 seedlings under various abiotic stresses by qRT-PCR. These results suggested that OsWRKY97 may be involved in responses to various stresses (Figure 1). For drought treatment, the transcription level of OSWRKY97 gradually increased until reaching a maximum at $8 \mathrm{~h}$ (Figure 1A). The transcription level of OsWRKY97 reached its maximum after treatment with $50 \mu \mathrm{M}$ ABA for 12 hours, and similar results were obtained after treatment with $250 \mathrm{mM}$ $\mathrm{NaCl}$ (Figure 1B,C). The expression of OsWRKY97 increased significantly in 12 to $24 \mathrm{~h}$ under cold stress (Figure 1D). However, the transcription level of OsWRKY97 under high temperature treatment was not significantly induced (Figure 1E).

\section{Subcellular localization of OsWRKY97}

To determine the subcellular localization of OsWRKY97, the full-length cDNA sequence of OsWRKY97 was fused to GFP and driven by CaMV35S. The fusion protein and green fluorescent protein (GFP) control were transiently expressed in tobacco cells via Agrobacterium infiltration, and meanwhile, the carrier of the red fluorescent protein nuclear Localization Signal - red fluorescent protein (NLS-RFP) connected with the nuclear localization signal is used as the nuclear localization control. The fluorescence signal of the fusion protein was located in the nucleus (Figure 2A), while the fluorescence signal of the GFP control was located in the nucleus and cytoplasm (Figure 2B). OsWRKY97-GFP fusion protein and NLS-RFP were co-located in the nucleus, indicating that OSWRKY97 is a nuclear protein.

\section{Overexpression of OsWRKY97 in rice improved osmotic stress tolerance at the germination and post- germination stages}

In this study, we found that OsWRKY97 was strongly induced by osmotic stress (Figure 1A). To further verify whether OsWRKY97 was involved in regulating the sensitivity of rice to stress, we constructed an OsWRKY97 overexpression vector and transferred it into Nipponbare. The expression level of OsWRKY97 in overexpressed plants was analyzed by qRT-PCR. The results showed that the expression of OsWRKY97 was significantly enhanced in transgenic lines (Figure $3 \mathrm{~A}$ ). Although we obtained $18 \mathrm{OE}$ lines, only two independent lines, $\mathrm{OE}-1$ and $\mathrm{OE}-23$ were selected for future testing. Under normal conditions, the germination rates of WT and OE lines were not significantly different. However, the germination rate of WT seeds was less than $50 \%$, however, after absorption for 5 days under the osmotic stress, the germination rate of overexpressed seed $\mathrm{OE}-1$ was $76 \%$, and that of $\mathrm{OE}-23$ was $80 \%$. (Figure 3B,C). To further understand the sensitivity of OE lines to osmotic stress at the post-germination stage, seedings of WT and OE lines that were growing under normal conditions for 4 days were selected and transferred to the nutrient solution with or without $20 \%$ PEG6000. After 12 days, each material was photographed, and 
its plant height was measured. Under normal conditions, the seedlings of WT and OE lines were similar in growth. Under osmotic stress, the plant height of WT seedlings was $8.6 \mathrm{~cm}$, while the plant height of overexpression seeding OE-1 was $10.8 \mathrm{~cm}$, and that of OE-23 was $10.6 \mathrm{~cm}$ (Figure 3D,E). These results showed that overexpression of OSWRKY97 in rice did not affect seed germination and seedling growth under normal growth conditions, but significantly attenuated the inhibitory effects on seed germination and seedling under osmotic stress induced by $20 \%$ PEG6000.

\section{Overexpression of OsWRKY97 in rice enhanced drought stress tolerance}

To further verify the biological function of OsWRKY97, we tested the drought stress tolerance of OE lines by withholding water. The seedings of OE lines and WT in the same pots that were of similar vigor were not watered until the leaves curled and then recovered. Before the pressure was introduced, the growth conditions of all genotypes were similar. After drought treatment, the leaf rolling degree of WT plants was more serious than that of OE plants. After re-watering, The survival rate of overexpressed plant OE- 1 was $53 \%$, and that of OE-23 was $55 \%$, however, the survival rate of WT was only $14 \%-16 \%$ (Figure $4 A, B$ ). These results indicated that overexpression of OsWRKY97 enhances the drought tolerance of rice. The relative water loss rate of detached leaves is an important characteristic reflecting drought tolerance[25]. Leaves of 2-week-old transgenic plants and WT seedlings were removed and exposed to water-free air for dehydration. Compared with WT, the leaf water loss of OE lines was significantly slower (Figure 4C), which means that OsWRKY97 played an active role in improving the water retention capacity of plants under dehydration conditions.

\section{Effects of OsWRKY97 overexpression on related physiological indexes under drought stress}

To further clarify the physiological mechanism by which OsWRKY97 confers tolerance to drought, we investigated the possible physiological basis related to the enhancement of drought resistance in $\mathrm{OE}$ plants. ROS were generated in plants when they were subjected to abiotic stresses such as drought, salinity, heat and cold[26]. $\mathrm{H}_{2} \mathrm{O}_{2}$, which is an important second messenger, is one of the most significant of these ROS[27]. Therefore, we measured the accumulation of $\mathrm{H}_{2} \mathrm{O}_{2}$ in plants after drought treatment. We found that the accumulation of $\mathrm{H}_{2} \mathrm{O}_{2}$ in OsWRKY97-overexpressing plants after drought treatment was much lower than that of WT (Figure 5A). To explore the potential mechanism of the reduction of active oxygen level in transgenic plants, ROS scavenging enzyme activity was also measured[11]. As shown in Figure 6, there was no significant difference in SOD, CAT and POD activities between transgenic lines and WT under normal conditions, while the enzyme activities of transgenic lines were higher than those of WT lines under drought stress (Figure 5B-D).Abiotic stresses to plants lead to enhanced membrane peroxidation and accumulation of osmotic substances in plants to maintain osmotic potential tissues of leaves[28]. As shown in Figure 5E, under drought conditions, the MDA content of transgenic plants and WT plants increased, but the increase of MDA content in transgenic plants was significantly lower than that of WT plants, which indicated that overexpression of OsWRKY97 in rice could reduce membrane lipid peroxidation under drought stress. Under drought stress, the proline content of transgenic plants was significantly higher than that of control plants (Figure 5F). This result clearly shows that 
overexpression of OsWRKY97 can increase proline synthesis and protect rice plants to better cope with drought stress.

\section{OsWRKY97 may be a positive regulator in ABA signaling under drought stress}

In this study, we found that OsWRKY97 was also strongly induced by ABA (Figure 1B), to verify this expression pattern, the sensitivities of OE plants to exogenous ABA were examined. The results showed that the germination rate of WT plants was higher than OE plants under the treatment of exogenous ABA (Figure $6 A, B$ ). Therefore, we suspect that the drought tolerance of $O E$ transgenic lines may be related to $A B A$. In order to verify this hypothesis, we measured the ABA content in OE lines and WT respectively under drought stress. The results showed that the endogenous ABA level was not significantly different between WT plants and OE plants under normal conditions. However, under drought stress, the endogenous ABA level in OE plants was significantly higher than WT plants (Figure 6C). In addition, we also analyzed the transcription level of response genes in ABA signaling pathway in WT plants and OE plants, including OsRAB21[OsRD22, OsRAB16A, OsNCED3[29, 30]. As shown in Figure 6D, the transcription level of these genes in OE plants was significantly higher than WT plants under drought stress. These results indicate that OsWRKY97 may improve drought tolerance via ABA signaling pathway.

\section{Discussion}

Plants will suffer from various abiotic stresses in their natural environment, which will affect the normal development of plants and even affect their yield[31,32]. At present, many studies have shown that WRKY TFs are negatively or positively involved in the integration of signaling pathways in abiotic stress responses[33]. However, the functions of many WRKY TFs in plants, especially stress responses, are still unclear. In this study, we examined whether OsWRKY97 participates in the regulation of the response to drought stress and its effect on rice. In addition, we found that OsWRKY97-GFP subcellular localization was in the nucleus of tobacco epidermal cells (Fig. 2), which is consistent with previous studies on other WRKY TFs and may be related to its function[34].

Increasing evidence shows that WRKY TFs play an important role in the drought stress response, as ectopic expression of OsWRKY11 enhanced tolerance to drought stress and induced constitutive expression of drought-responsive genes[35]. Overexpression of GsWRKY20 from Glycine soja L.G07256 in Arabidopsis resulted in increased sensitivity to ABA when stomata were closed and stronger drought tolerance compared with the WT[36]. Similarly, our results showed that OsWRKY97 expression was rapidly activated under drought stress, suggesting that OsWRKY97 may play an important role in drought stress. Therefore, we constructed OE transgenic plants and tested their resistance to drought stress. The results showed that the germination rate of $\mathrm{OE}$ at the germination stage was significantly higher, and the growth inhibition of OsWRKY97 at the seedling stage was also weakened compared with that of WT plants under osmotic stress (Fig. 3). These results suggest that OsWRKY97 may positively regulate drought stress tolerance. In addition, this conclusion was supported by the result that the survival rate of OE plants was higher than that of WT plants under drought conditions (Fig. 4A,B). 
Plants respond to water loss at physiological, cellular and molecular levels[37]. ABA is an important plant hormone involved in plant developmental process,which is widely considered as the main regulatory factor of plant response to drought. ABA reduces water loss by inducing stomatal closure and induces a number of stress response genes[38, 39]. It has been reported that ABA-independent and ABA-dependent regulatory systems both exist in response to drought stress[40]. In this study, First, we found that OsWRKY97 was strongly induced by exogenous ABA (Fig. 1B). Second, the sensitivities of OE plants to exogenous ABA was enhanced (Fig. 6C). Third, the expression level of OsWRKY97 induces ABA accumulation and the expression level of $A B A$ responsive gene under drought stress (Fig. 6A,D), this result was consistent with the capability of OsWRKY97 to reduce the water loss rate of plants under drought conditions (Fig. 4C). These results intimate that OsWRKY97 improves drought tolerance by enhancing water retention of rice through ABA-dependent pathway. Meanwhile the up-regulation of OsWRKY97 expression under drought stress will lead to the up-regulation of ABA biosynthesis and response genes, resulting in $A B A$ accumulation and increased sensitivity to exogenous $A B A$.

$\mathrm{H}_{2} \mathrm{O}_{2}$ is not only an important ROS but also the pivot for the mutual conversion of ROS, which is also an important signal molecule at normal levels[27]. $A B A$ signal interacts with $\mathrm{H}_{2} \mathrm{O}_{2}$ in plant tissues. There is evidence that $\mathrm{H}_{2} \mathrm{O}_{2}$ acts upstream of $A B A$ signaling pathway. Exogenous $\mathrm{H}_{2} \mathrm{O}_{2}$ increases $A B A$ catabolism during seed germination by enhancing the expression of CYP707A genes [41, 42]. It has also been reported that $\mathrm{H}_{2} \mathrm{O}_{2}$ plays an important role as a second messenger in ABA-induced stomatal closure in guard cells $[8,9]$. It is reported that $\mathrm{H}_{2} \mathrm{O}_{2}$ can be induced by $A B A$, which is mediated by inducing plant gene expression encoding NADPH oxidase to respond to $A B A[43,44]$. However, the increase of $\mathrm{H}_{2} \mathrm{O}_{2}$ content induced by drought stress is more obvious than that caused by exogenous $A B A$, this shows that the water deficit signal enhances the production of ROS to a greater extent, which will pose a threat to plants[45]. As expected, the level of $\mathrm{H}_{2} \mathrm{O}_{2}$ in plants has increased under drought stress. However, the accumulation of $\mathrm{H}_{2} \mathrm{O}_{2}$ in WT plants was significantly higher than that in OE plants (Fig. 5A), and the activities of major oxygen scavenging enzymes, including SOD, POD, CAT, were also observably higher than those in $\mathrm{OE}$ plants (Fig. 5B-D). SOD, POD and CAT, which maintain the ROS homeostasis, were activated in OE plants.

As a product of ROS-stimulated lipid peroxidation, MDA contents can be used to evaluate the extent of ROS-mediated injuries in plants. In our research, we found that ROS in WT plants accumulated so much that MDA content increased correspondingly compared with OE plants under drought stress (Fig. 5E). In addition, we also found that drought stress led to the accumulation of osmotic compound, proline, in OE plants was more than WT plants (Fig. 5F). Under osmotic conditions, proline, as an important osmotic protective agent, can maintain low water potential of cells so as not to be damaged by active enzymes[46]. These results showed that WT was more seriously damaged than OE plants under osmotic conditions, thus increasing the probability of WT death.

\section{Conclusions}


In conclusion, we identified OsWRKY97, a transcription factor located in the nucleus. OsWRKY97 affects the sensitivity of rice to exogenous ABA and the accumulation of ABA content. In addition, OsWRKY97 affects the redox balance and drought resistance of rice. Redox related mechanisms might be involved in OsWRKY97 mediated drought tolerance, which might affect the content of proline and MDA in rice. All these results indicated that OsWRKY97 gene has a high potential in improving rice drought resistance.

\section{Materials And Methods}

\section{Plant material and growth conditions}

Japonica rice (Oryza sativa L. japonica. cv. Nipponbare) was selected as the WT in this study. Rice seeds were sterilized with $0.1 \% \mathrm{NaClO}$ for 30 min before being soaked in distilled water for 2 days in the dark and were then transferred to a culture dish containing Hoagland nutrient solution and grown in a climate chamber (Southeast instrument, Ningbo, China) with the temperature of $28^{\circ} \mathrm{C}$, the relative humidity of 70\%,and the 14-hour light/10-hour dark photoperiod[47].

\section{Abiotic treatments}

To determine the expression pattern of OsWRKY97 under different stress conditions, two-week-old seedlings of Nipponbare rice were subjected to various stress treatments. For drought treatment, seedlings were grown in culture solution containing $20 \% \mathrm{PEG} 6000$. For salt treatment, $\mathrm{NaCl}$ solution was added to achieve a final concentration of $250 \mathrm{mM}$. ABA treatment was carried out by adding $50 \mu \mathrm{M} \mathrm{ABA}$ to the culture solution. The seedlings were transferred to a $4^{\circ} \mathrm{C}$ climate chamber for cold treatment. For heat stress, the seedlings were subjected to $42^{\circ} \mathrm{C}$ heat shock treatment[48, 49]. Samples were collected at $0,1,2,4,8,12,16$ and $24 \mathrm{~h}$ after treatment. Two-week-old seedings of OsWRKY97 overexpression lines were subjected to drought treatment, and samples were collected at $0 \mathrm{~h}$ and $10 \mathrm{~h}$ after treatment respectively.

\section{RNA extraction and real-time PCR}

The leaves of 14-day-old plants were sampled, and total RNA was extracted with TRIzol reagent (Invitrogen, Nanjing, China) according to the manufacturer's instructions. One microgram of DNasetreated RNA was reverse-transcribed using a RevertAid RT Reverse Transcription Kit (TaKaRa, Beijing, China) according to the manufacturer's protocol. Real-time PCR was performed on a Bio-Rad CFX96 realtime PCR system. Each reaction was performed in triplicate, and the reaction procedure was as follows: $95^{\circ} \mathrm{C}$ for $10 \mathrm{~min}$ and, then $39 \mathrm{cycles}$ of $95^{\circ} \mathrm{C}$ for $10 \mathrm{~s}$ and, $60^{\circ} \mathrm{C}$ for $30 \mathrm{~s}$. The data of relative expression level were analyzed by the $2^{-\triangle \Delta \mathrm{Ct}}$ method[50]. The OsActin rice gene was used as an internal control gene, and relevant primer pairs are listed in Additional file 2:Table S1.

\section{Vector construction and gene transformation}


To construct the overexpression vector of OsWRKY97, cDNA from rice (Oryza sativa L. japonica. cv. Nipponbare) was used. Total RNA was used to amplify the open reading frame of OsWRKY97, and the relevant primer pairs are listed in Additional file 2:Table S1. The fragments confirmed by sequencing were digested and cloned into the PCAMBIA1300 vector, and the digestion sites were HindIII and Sall. This gene was driven by the CaMV35S promoter (Additional file 3:Figure S2). All the vectors were transferred into Nipponbare plants via Agrobacterium-mediated transformation[51].

\section{Determination of stress-associated physiological indicators}

Seedlings growing under normal conditions for two weeks were transferred to a solution with or without $20 \%$ PEG6000. Samples were collected at $0 \mathrm{~h}$ and $10 \mathrm{~h}$, and then, the corresponding physiological indexes were measured. MDA content was qualified by thecreaction method as described by Gao[52]. $\mathrm{H}_{2} \mathrm{O}_{2}$ content was determined according to the method of Zhan[53]. For the water loss rate, two-week-old seedlings were selected, exposed to air under the condition of no water supply and weighed and recorded every half hour until the weight was constant. Water loss rate was calculated by comparing the measured weight from each indicated time with the measurement at time zero. It is expressed as percentage of initial fresh weight[54]. Proline was detected by following the reported methods[55]. The activity of active oxygen scavenging enzymes was determined according to previous methods[56].

\section{Measurement of ABA content}

We analyzed the content of ABA in rice by liquid chromatography-mass spectrometry (LC-MS)[57]. Briefly, $1 \mathrm{~g}$ of frozen leaf tissue was extracted in $10 \mathrm{ml}$ of acetone/water/acetic acid (80:19:1, v/v). The complete homogenate was incubated overnight in darkness at $4^{\circ} \mathrm{C}$. After that, they were vortexed and centrifuged at $15,000 \mathrm{rpm}, 4^{\circ} \mathrm{C}$ for 10 minutes, and the crude extract supernatant was collected and dried in in a rotavapor until an aqueous fractions were obtained. Dried samples were resuspended in $1 \mathrm{~mL}$ of acetonitrile/water/acetic acid (90:10:0.05, v/v), and filtered through a $0.45 \mu \mathrm{m}$ PTFE filter (JET BIOFIL, Guangzhou, China). Quantification was done by the standard addition method by spiking control plant samples with ABA solutions.

\section{Subcellular localization of OsWRKY97}

The coding region of OsWRKY97 was amplified and cloned into the pAcGFP1-N1 vector to generate the OsWRKY97-GFP (green fluorescent protein) fusion construct, which was inserted into the pCAMBIA1300 vector, and the constructed vector was transferred into Agrobacterium. After sequencing confirmation, the fusion structure and the control (empty body) vector were co-transfected with another NLS-RFP vector respectively into tobacco cells by Agrobacterium mediated transient expression method, and the fluorescence signal was observed by confocal laser scanning microscope[58].

\section{Analysis of stress tolerance}


Two independent T2 over-expression lines and WT seeds were soaked in clear water for two days after being sterilized by $0.1 \% \mathrm{NaClO}$, and then, the seeds were transferred to culture solution for growth. When the plant height was approximately $1 \mathrm{~cm}$, seedlings with similar growth potential were selected and transferred to nutrient solution containing $20 \% \mathrm{PEG}$, and their growth conditions were observed and recorded. To simulate an arid environment, the two-week-old seedlings were transferred to the sand, and watering was stopped after two days of normal irrigation. The mortality rates were determined. In order to test the sensitivity of ABA sensitivity at germination stage, seeds of OE plants and WT plants were germinated on culture solution containing $5 \mu \mathrm{M} \mathrm{ABA}$, and the germination rate was calculated on the 6 th day after germination.

\section{Statistical Analysis}

Results are reported as the mean \pm standard deviation (SD) values of the three independent biological experiments, all experiments were repeated at least three times, Statistical analysis was performed using the Student's t-test by SPSS 18.0 (Chicago, IL) sofware package, $* p<0.05, * * p<0.01$.

\section{Abbreviations}

ABA

abscisic acid

WT

wild-type

MDA

malondialdehyde

ROS

reactive oxygen species

SOD

superoxide dismutase

CAT

catalase

POD

peroxidase

OE

OsWRKY97-overexpressing

GFP

green fluorescent protein

NLS-RFP

Nuclear Localization Signal - red fluorescent protein

PYR

Pyrabactin resistance

PYL 
PYR1-like

RCARs

Regulatory component of ABA receptors

PP2C

2C-type protein phosphatase

SnRK2

SNF1-related protein kinase 2

TF

Transcription factor

AREB

ABRE-binding protein

ABRE

ABA-responsive element

qRT-PCR

quantitative real-time RT-PCR

\section{Declarations}

\section{Contributions}

ML, DH, TY, LZ, JF, JX, CL and LC carried out the experiments. SR, YD, WC, YS, ZH and JZ analyzed the data $L L$ and $Z X$ conceived and designed the research. $L L, M L$ and $X G$ wrote and revised the manuscript. All authors read and approved the final manuscript.

\section{Acknowledgements}

This work was supported by the International Cooperation Project of Sichuan Provincial Science and Technology Department under grant number $2017 \mathrm{HH} 0031$ and the Fundamental Research Funds for the Central Universities under grant number 2662017JC026.

\section{Funding}

This work was supported by the International Cooperation Project of Sichuan Provincial Science and Technology Department under grant number 2017HH0031 and the Fundamental Research Funds for the Central Universities under grant number 2662017JC026.

\section{Availability of data and materials}

All data analyzed during this study are included in this published article and its supplementary information files.

\section{Competing interests}


We declare that we have no conflict of interest.

\section{Ethics approval and consent to participate}

Not applicable.

\section{Consent for publication}

Not applicable.

\section{References}

1. Mickelbart MV, Hasegawa PM, Bailey-Serres J: Genetic mechanisms of abiotic stress tolerance that translate to crop yield stability. Nat Rev Genet. 2015; 16:237-251.

2. Fang $Y$, Xiong $L$ : General mechanisms of drought response and their application in drought resistance improvement in plants. Cell Mol Life. 2015;72:673-689.

3. Xiong L, Schumaker KS, Zhu JK: Cell signaling during cold, drought, and salt stress. Plant Cell . 2002;14:S165-S183.

4. Zhu JK: Salt and drought stress signal transduction in plants. Annu Rev Plant Biol. 2002;53:247-273.

5. Hubbard KE, Nishimura N, Hitomi K, Getzoff ED, Schroeder JI: Early abscisic acid signal transduction mechanisms: newly discovered components and newly emerging questions. Gene Dev. 2010;24:1695-1708.

6. Mizuno T, Yamashino T: Comparative transcriptome of diurnally oscillating genes and hormoneresponsive genes in Arabidopsis thaliana: insight into circadian clock-controlled daily responses to common ambient stresses in plants. Plant Cell Physiol. 2008;49:481-487.

7. Desikan. R, Cheung M-K, Bright J, Henson D, Hancock JT, Neill SJ: ABA, hydrogen peroxide and nitric oxide signalling in stomatal guard cells. J Exp Bot. 2004;55:205-212.

8. Hamilton DW, Hills A, Kohler B, Blatt MR: $\mathrm{Ca}^{2+}$ channels at the plasma membrane of stomatal guard cells are activated by hyperpolarization and abscisic acid. P Natl Acad Sci USA. 2000;97:4967-4972.

9. Zhang X, Zhang L, Dong F, Gao J: Hydrogen Peroxide Is Involved in Abscisic Acid-Induced Stomatal Closure in Vicia faba. Plant Physiol. 2001;126:1438-1448.

10. Liu J, Zhang C, Wei C, Liu X, Wang M, Yu F, Xie Q, Tu J: The RING Finger Ubiquitin E3 Ligase OsHTAS Enhances Heat Tolerance by Promoting H2O2-Induced Stomatal Closure in Rice. Plant Physiol. 2016;170:429-443.

11. Apel K, Hirt H: REACTIVE OXYGEN SPECIES: Metabolism, Oxidative Stress, and Signal Transduction. Annu Rev Plant Biol. 2004;55373-399.

12. Deb A, Kundu S: Deciphering Cis-Regulatory Element Mediated Combinatorial Regulation in Rice under Blast Infected Condition. PloS One. 2015;10:e0137295. 
13. Song H, Sun W, Yang G, Sun J: WRKY transcription factors in legumes. BMC Plant Biol. 2018; 18:243256.

14. Chi Y, Yang Y, Zhou Y, Zhou J, Fan B, Yu JQ, Chen Z: Protein-protein interactions in the regulation of WRKY transcription factors. Mol Plant. 2013;6:287-300.

15. Gao H, Wang Y, Xu P, Zhang Z: Overexpression of a WRKY Transcription Factor TaWRKY2 Enhances Drought Stress Tolerance in Transgenic Wheat. Front Plant Sci. 2018;9:997.

16. Joshi R, Wani SH, Singh B, Bohra A, Dar ZA, Lone AA, Pareek A, Singla-Pareek SL: Transcription Factors and Plants Response to Drought Stress: Current Understanding and Future Directions. Front Plant Sci. 2016;7:1029.

17. Jaffar M, Song A, Faheem M, Chen S, Jiang J, Liu C, Fan Q, Chen F: Involvement of CmWRKY10 in Drought Tolerance of Chrysanthemum through the ABA-Signaling Pathway. Int J Mol Sci. 2016;17:693.

18. Tao Z, Kou Y, Liu H, Li X, Xiao J, Wang S: OsWRKY45 alleles play different roles in abscisic acid signalling and salt stress tolerance but similar roles in drought and cold tolerance in rice. J Exp Bot. 2011;62:4863-4874.

19. Yoshida T, Fujita Y, Sayama H, Kidokoro S, Maruyama K, Mizoi J, Shinozaki K, Yamaguchi-Shinozaki $\mathrm{K}$ : AREB1, AREB2, and ABF3 are master transcription factors that cooperatively regulate ABREdependent $A B A$ signaling involved in drought stress tolerance and require $A B A$ for full activation. Plant J. 2010;61:672-685.

20. Nakashima K, Yamaguchi-Shinozaki K: ABA signaling in stress-response and seed development. Plant Cell Rep. 2013;32:959-970.

21. Fujii $\mathrm{H}$, Zhu JK: Arabidopsis mutant deficient in 3 abscisic acid-activated protein kinases reveals critical roles in growth, reproduction, and stress. Plant Cell Rep. 2009;106:8380-8385.

22. Antoni R, Rodriguez L, Gonzalez-Guzman M, Pizzio GA, Rodriguez P: News on ABA transport, protein degradation and ABFs/WRKYs in ABA signaling. Curr Opin Plant Biol. 2011;14:547-553.

23. Ren X, Chen Z, Liu Y, Zhang H, Zhang M, Liu Q, Hong X, Zhu JK, Gong Z: ABO3, a WRKY transcription factor, mediates plant responses to abscisic acid and drought tolerance in Arabidopsis. Plant $\mathrm{J}$. 2010;63:417-429.

24. Zhi-Qiang Liu, Lu Yan, Zhen Wu, Chao Mei, Kai Lu: Cooperation of three WRKY-domain transcription factors WRKY18, WRKY40, and WRKY60 in repressing two ABA-responsive genes ABI4 and ABI5 in Arabidopsis. J Exp Bot. 2012;63:6371-6392.

25. Yao L, Cheng X, Gu Z, Huang W, Li S, Wang L, Wang YF, Xu P, Ma H, Ge X: The AWPM-19 Family Protein OsPM1 Mediates Abscisic Acid Influx and Drought Response in Rice. Plant Cell. 2018; 30:1258-1276.

26. Miller GAD, Mittler RON: Could Heat Shock Transcription Factors Function as Hydrogen Peroxide Sensors in Plants? Ann Bot. 2006;98:279-288.

27. Zhao Q, Zhou L, Liu J, Du X, Asad MA, Huang F, Pan G, Cheng F: Relationship of ROS accumulation and superoxide dismutase isozymes in developing anther with floret fertility of rice under heat stress. 
Plant physiol Bioch. 2018;122:90-101.

28. Hasegawa PM, Bressan RA: Plant cellular and molecular responses ro high salinity. Annu Rev Plant Bio. 2000;51:463-499.

29. Huang Y, Guo Y, Liu Y, Zhang F, Wang Z, Wang H, Wang F, Li D, Mao D, Luan S et al: 9-cisEpoxycarotenoid Dioxygenase 3 Regulates Plant Growth and Enhances Multi-Abiotic Stress Tolerance in Rice. Front Plant Sci. 2018;9:162.

30. Mundy J, Chua $\mathrm{N}-\mathrm{H}$ : Abscisic acid and water-stress induce the expression of a novel rice gene. EMBO J. 1988;7:2279-2286.

31. Wang W, Vinocur B, Altman A: Plant responses to drought, salinity and extreme temperatures: towards genetic engineering for stress tolerance. Planta. 2003;218:1-14.

32. Bohnert HJ, Gong Q, Li P, Ma S: Unraveling abiotic stress tolerance mechanisms--getting genomics going. Curr Opin Plant Biol. 2006;9:180-188.

33. Zou C, Jiang W, Yu D: Male gametophyte-specific WRKY34 transcription factor mediates cold sensitivity of mature pollen in Arabidopsis. J Exp Bot. 2010;61:3901-3914.

34. Shen H, Liu C, Zhang Y, Meng X, Zhou X, Chu C, Wang X: OsWRKY30 is activated by MAP kinases to confer drought tolerance in rice. Plant Mol Biol. 2012;80:241-253.

35. Lee H, Cha J, Choi C, Choi N, Ji HS, Park SR, Lee S, Hwang DJ: Rice WRKY11 Plays a Role in Pathogen Defense and Drought Tolerance. Rice. 2018;11:5.

36. Luo X, Bai X, Sun X, Zhu D, Liu B, Ji W, Cai H, Cao L, Wu J, Hu M et al: Expression of wild soybean WRKY20 in Arabidopsis enhances drought tolerance and regulates ABA signalling. J Exp Bot. 2013; 64:2155-2169.

37. Shinozaki K, Yamaguchi-Shinozaki K: Gene networks involved in drought stress response and tolerance. J Exp Bot. 2006;58:221-227.

38. Fujita Y, Fujita M, Shinozaki K, Yamaguchi-Shinozaki K: ABA-mediated transcriptional regulation in response to osmotic stress in plants. J plant Res. 2011;124:509-525.

39. Leon J, Castillo MC, Coego A, Lozano-Juste J, Mir R: Diverse functional interactions between nitric oxide and abscisic acid in plant development and responses to stress. J Exp Bot. 2014; 65:907-921.

40. Yamaguchi-Shinozaki K, Shinozak K: Transcriptional Regulatory Networks in Cellular Responses and Tolerance to Dehydration and Cold Stresses. Annu Rev Plant Biol. 2006;57:781-803.

41. Liu Y, Ye N, Liu R, Chen M, Zhang J: H2O2 mediates the regulation of ABA catabolism and GA biosynthesis in Arabidopsis seed dormancy and germination. J Exp Bot. 2010;61:2979-2990.

42. Saxena I, Srikanth S, Chen Z: Cross Talk between H2O2 and Interacting Signal Molecules under Plant Stress Response. Front Plant Sci. 2016;7:570.

43. JM K, IC M, ZM P, N L, MA T, JL D: NADPH oxidase AtrbohD and AtrbohF genes function in ROSdependent ABA signaling in Arabidopsis. EMBO J. 2003;22:2623-2633.

44. Ozfidan C, Turkan I, Sekmen AH, Seckin B: Abscisic acid-regulated responses of aba2-1 under osmotic stress: the abscisic acid-inducible antioxidant defence system and reactive oxygen species 
production. Plant Biol. 2012;14:337-346.

45. Phillips K, Ludidi N: Drought and exogenous abscisic acid alter hydrogen peroxide accumulation and differentially regulate the expression of two maize RD22-like genes. Sci Rep. 2017; 7:8821.

46. Kakar KU, Ren XL, Nawaz Z, Cui ZQ, Li B, Xie GL, Hassan MA, Ali E, Sun GC: A consortium of rhizobacterial strains and biochemical growth elicitors improve cold and drought stress tolerance in rice (Oryza sativa L.). Plant Biol. 2016;18:471-483.

47. Yoshida S, Forno DA, Cock JH, Gomez KA: Laboratory Manual for Physiological Studies of Rice. Manila: IRRI. 1976;p:61-67.

48. Saijo Y, Hata S, Kyozuka J, Shimamoto K, Izui K: Over-expression of a single Ca2+-dependent protein kinase confers both cold and salt/drought tolerance on rice plants. Plant J. 2000; 23:319-327.

49. Gao Y, Wu M, Zhang M, Jiang W, Ren X, Liang E, Zhang D, Zhang C, Xiao N, Li Y et al: A maize phytochrome-interacting factors protein ZmPIF1 enhances drought tolerance by inducing stomatal closure and improves grain yield in Oryza sativa. Plant Biotechnol J. 2018;16:1375-1387.

50. Livak KJ, Schmittgen TD: Analysis of Relative Gene Expression Data Using Real-Time Quantitative PCR and the 2- $\Delta \Delta C T$ Method. Methods. 2001;25:402-408.

51. Ozawa K: A High-Efficiency Agrobacterium-Mediated Transformation System of Rice (Oryza sativa L.). Method Mol Biol. 2012;847:51-57.

52. Du H, Wang N, Cui F, Li X, Xiao J, Xiong L: Characterization of the beta-carotene hydroxylase gene DSM2 conferring drought and oxidative stress resistance by increasing xanthophylls and abscisic acid synthesis in rice. Plant Physiol. 2010;154:1304-1318.

53. Li Z, Gao Y, Lin C, Pan R, Ma W, Zheng Y, Guan Y, Hu J: Suppression of LOX activity enhanced seed vigour and longevity of tobacco (Nicotiana tabacum L.) seeds during storage. Conserv Physiol. 2018; 6:coy047.

54. Rong W, Qi L, Wang A, Ye X, Du L, Liang H, Xin Z, Zhang Z: The ERF transcription factor TaERF3 promotes tolerance to salt and drought stresses in wheat. Plant Biotechnol J.2014;12:468-479.

55. María Noelia Muñiz García, Juan Ignacio Cortelezzi, Marina Fumagalli, Daniela A. Capiati: Expression of the Arabidopsis ABF4 gene in potato increases tuber yield, improves tuber quality and enhances salt and drought tolerance. Plant Mol Biol. 2018;98:137-152.

56. Li M, Xu G, Xia X, Wang M, Yin X, Zhang B, Zhang X, Cui Y: Deciphering the physiological and molecular mechanisms for copper tolerance in autotetraploid Arabidopsis. Plant Cell Rep. 2017; 36:1585-1597.

57. Lopez-Carbonell M, Jauregui O: A rapid method for analysis of abscisic acid (ABA) in crude extracts of water stressed Arabidopsis thaliana plants by liquid chromatography-mass spectrometry in tandem mode. Plant Physiol Bioch. 2005;43:407-411.

58. He X, Li L, Xu H, Xi J, Cao X, Xu H, Rong S, Dong Y, Wang C, Chen R et al: A rice jacalin-related mannose-binding lectin gene, OsJRL, enhances Escherichia coli viability under high salinity stress and improves salinity tolerance of rice. Plant Biology. 2017;19:257-267. 
Figures

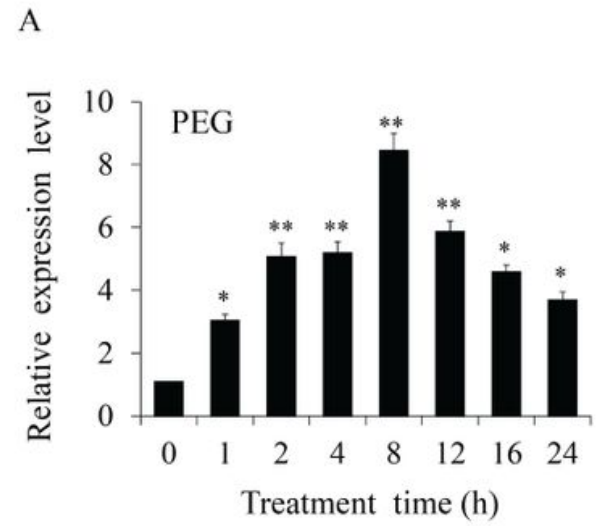

D

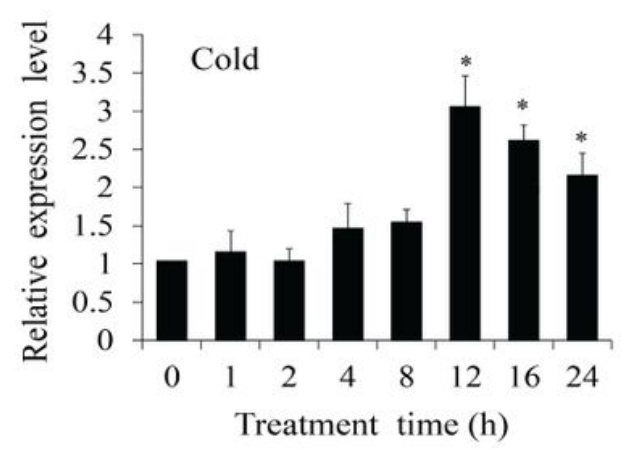

B

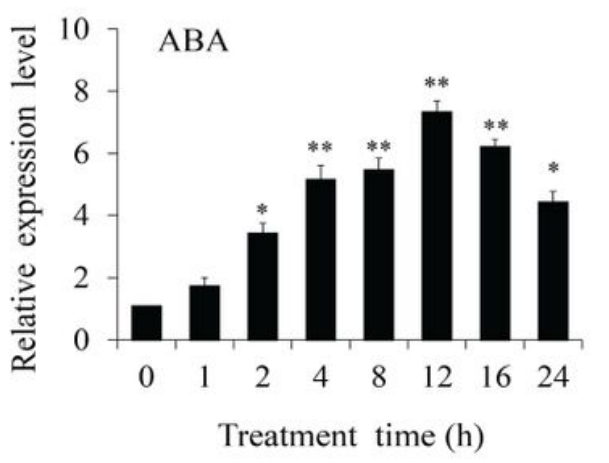

E

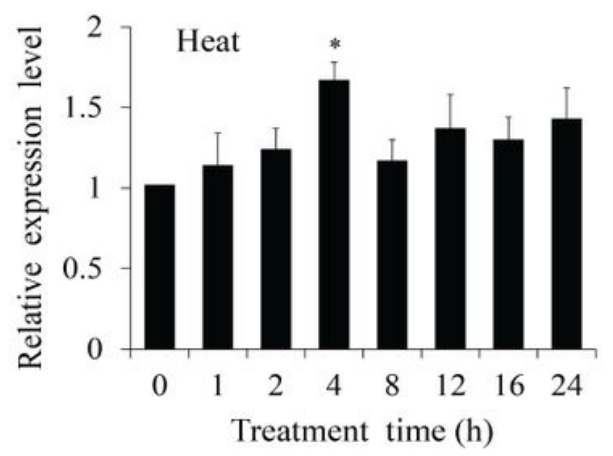

C

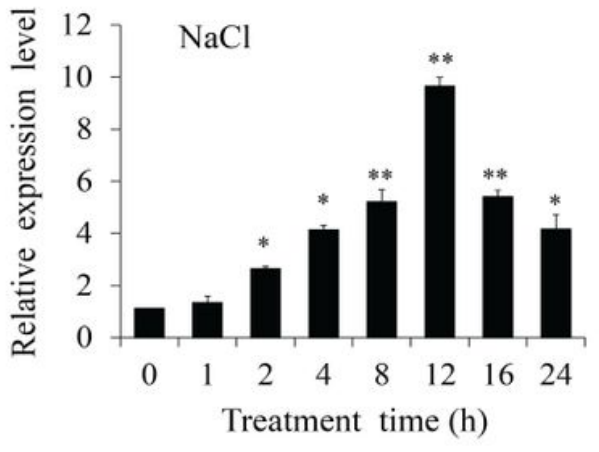

\section{Figure 1}

Expression analysis of the OsWRKY97 gene in rice. (A-E) OsWRKY97 expression analysis (qRT-PCR) in leaves of 2-week-old rice seedlings subjected to 20\% PEG6000, $50 \mu \mathrm{M} \mathrm{ABA}, 250 \mathrm{mM} \mathrm{NaCl}, \mathrm{cold}^{\circ}\left(4^{\circ} \mathrm{C}\right)$ and heat $\left(42^{\circ} \mathrm{C}\right)$ treatments, respectively. OsActin gene was used as internal controls. The data are represented as the mean $\pm S D(n=3)$, with three biological experiments. Asterisks indicates a significant difference from the value at 0 h (T-test, $* \mathrm{p}<0.05, * * \mathrm{p}<0.01)$. 
GFP
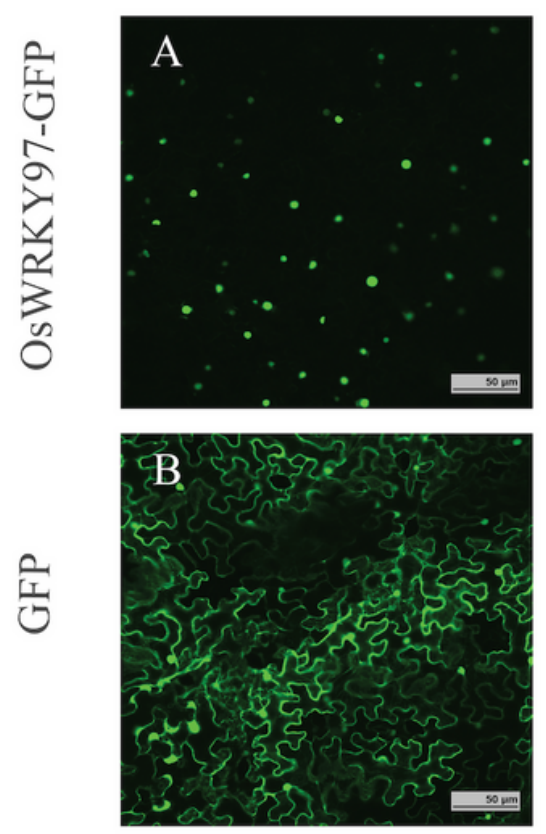

RFP
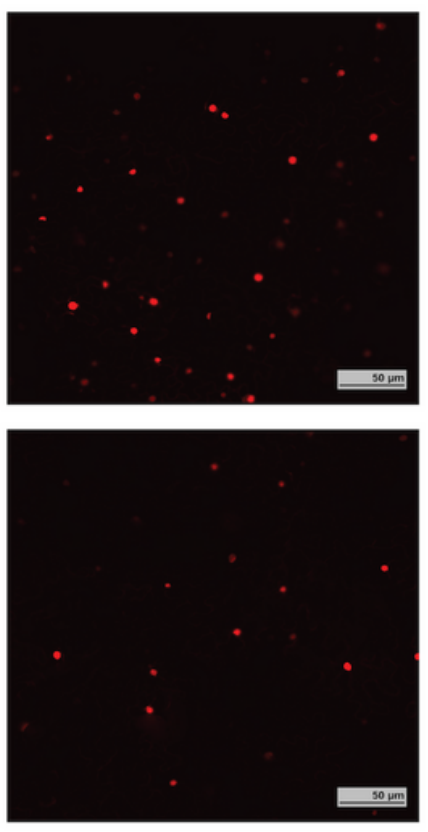

Bright
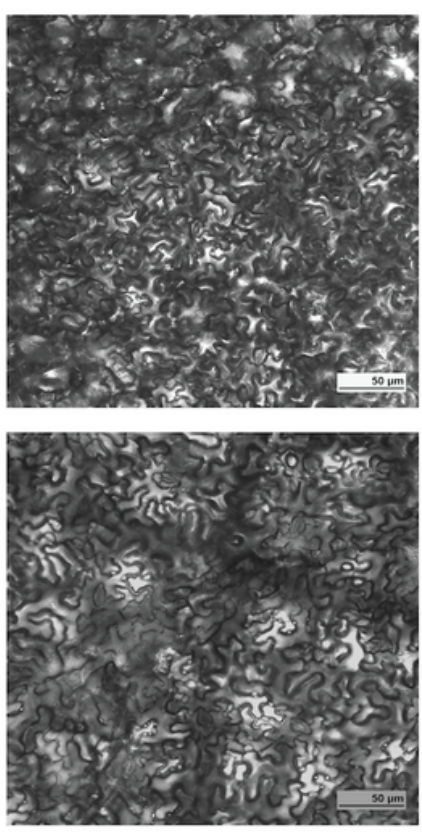

Merged
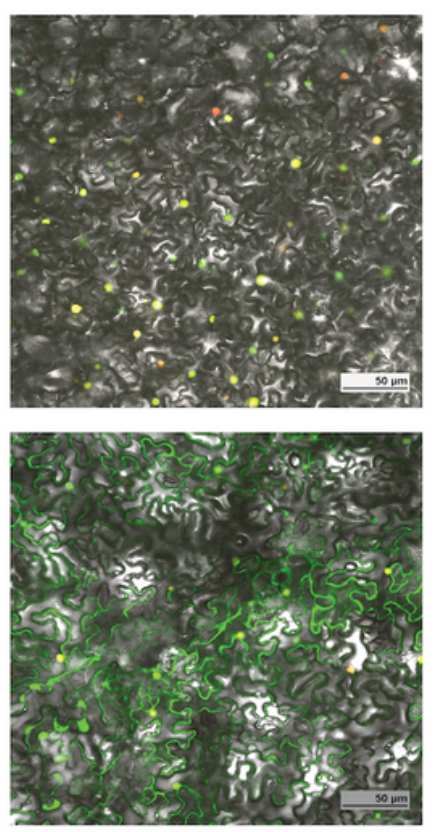

\section{Figure 2}

Subcellular localization of OsWRKY97-GFP. OsWRKY97 driven by the Cauliflower mosaic virus 35S promoter was transiently expressed in tobacco leaf epidermal cells and viewed with confocal microscopy. Nuclear and cytosolic localization of GFP protein was shown as a control. (A) 35S:OsWRKY97-GFP; (B) 35S: GFP. A vector harboring 35S::RFP::NLS (the RFP red fluorescent protein linked to a nuclear localization signal) was used as nucleus marker in transient co-expression assays. Bar $=50 \mu \mathrm{m}$. 
A

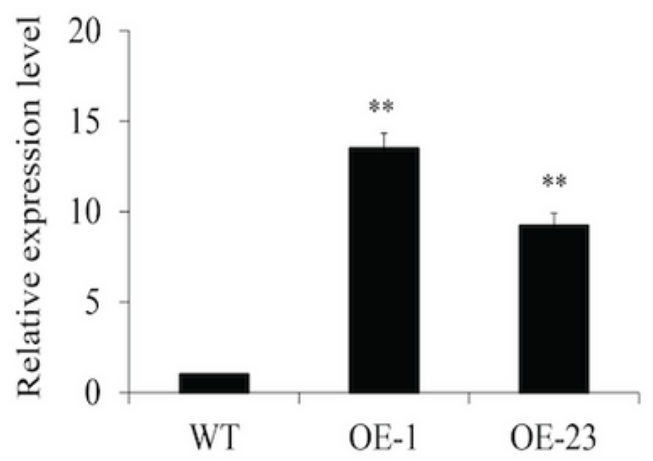

B

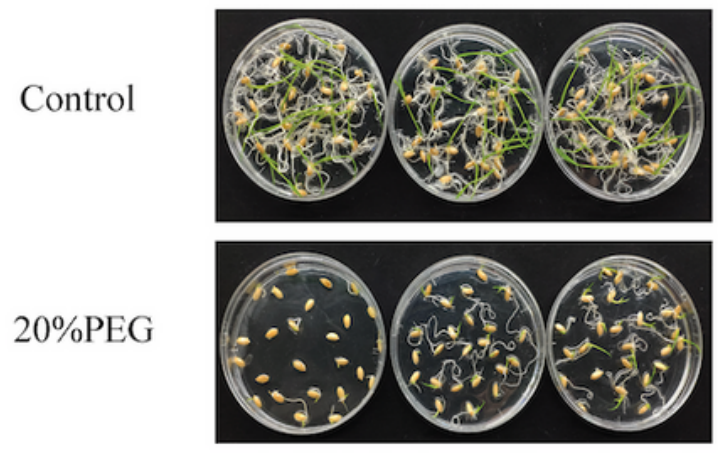

WT OE-1 OE-23

C

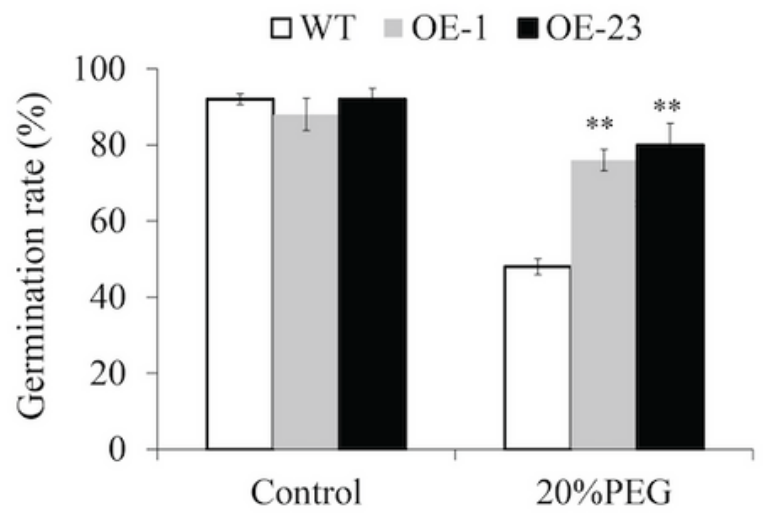

D

Control

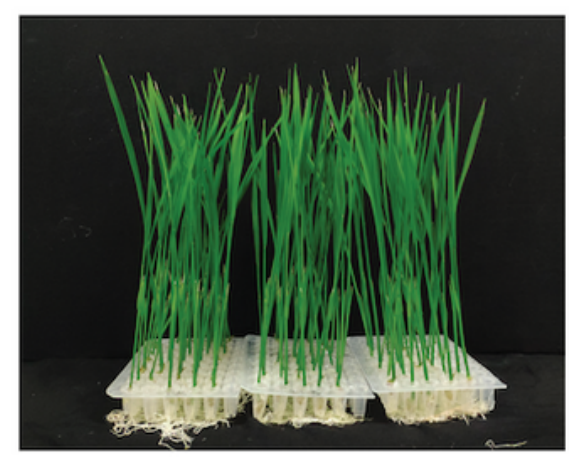

$20 \%$ PEG

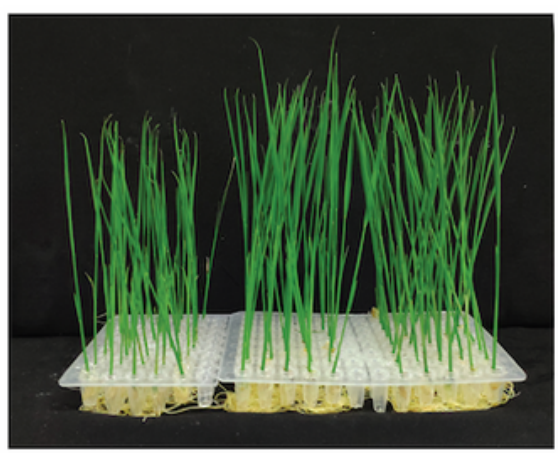

WT OE-1 OE-23

E

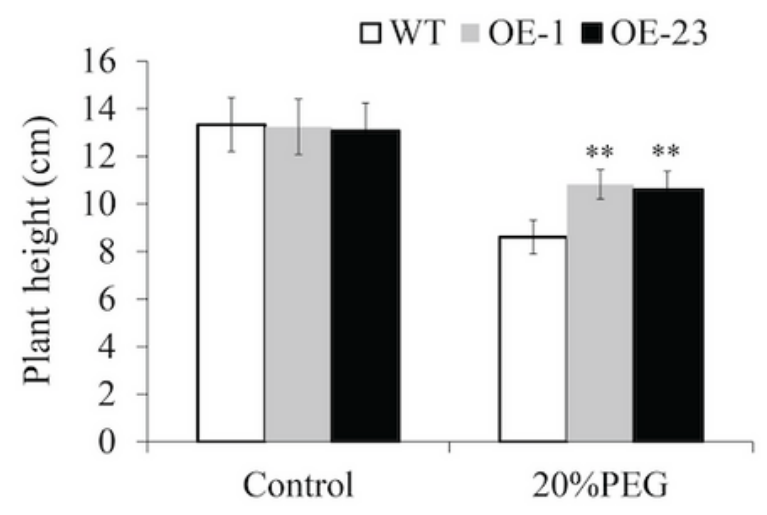

\section{Figure 3}

Phenotype of OsWRKY97-overexpressing plants under osmotic stress. (A) Two independent transgenic lines (OE-1, OE-23) of OsWRKY97 were verified by qRT-PCR. OsActin was analyzed as an internal control. Data were means \pm SD with at least three biological replicates. Asterisks represent statistically significant differences between WT and OE lines (T-test, $* p<0.05, * * p<0.01$ ). The growth performance (B) and germination rate (C) of OE lines and wild-type at 5 days after germination in nutrient solution with or 
without 20\% PEG6000. The data are represented as the mean \pm SD with at least three biological replicates and every replicate contains 20 individual plants. The growth phenotype (D) and plant height (E) of overexpression lines and WT, which were growing under normal conditions for 4 days, were transferred to nutrient solution with or without $20 \%$ PEG6000 for 12 days. Data are shown as the mean \pm $S D(n=4)$ with four biological replicates and every replicate contains 40 individual plants. The asterisk represents the statistically significant difference between the WT and OE lines under osmotic stress (Ttest, $* \mathrm{p}<0.05, * * \mathrm{p}<0.01)$.

A
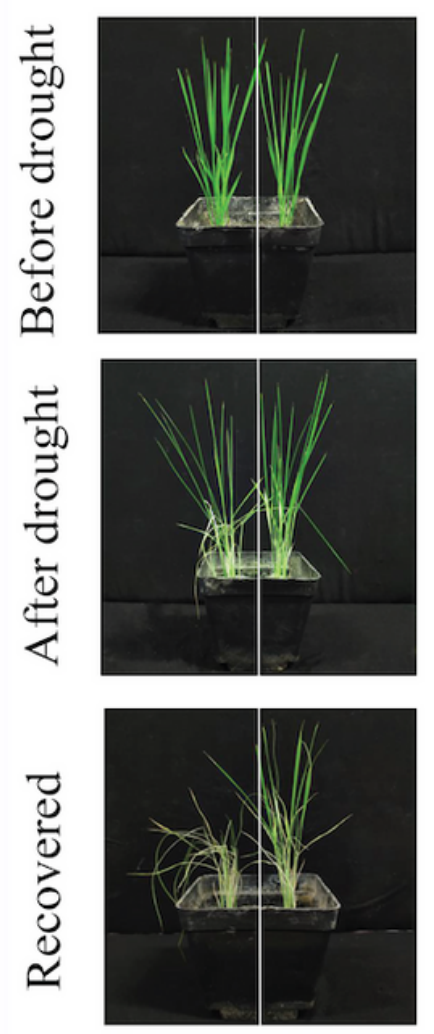

WT OE-1
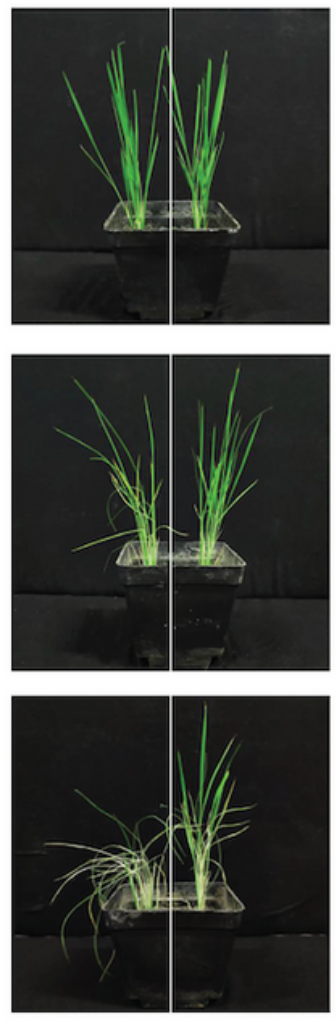

WT OE-23
B

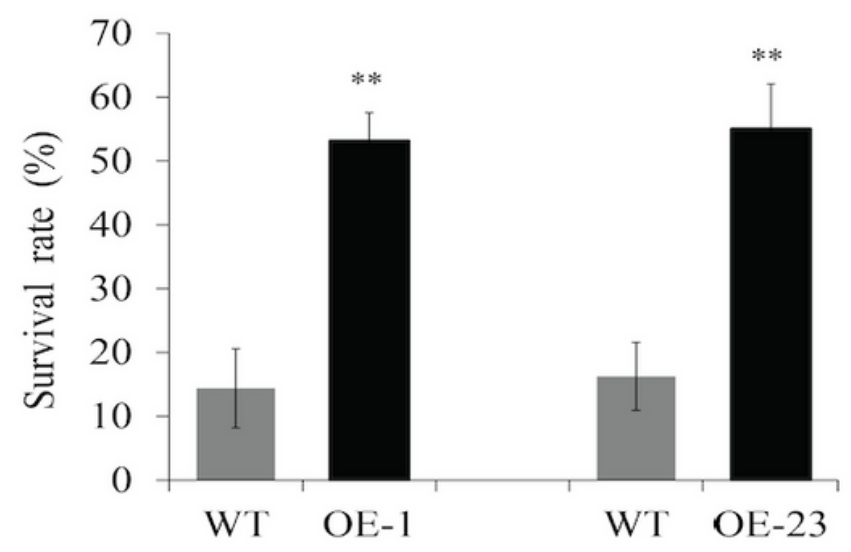

$\mathrm{C}$

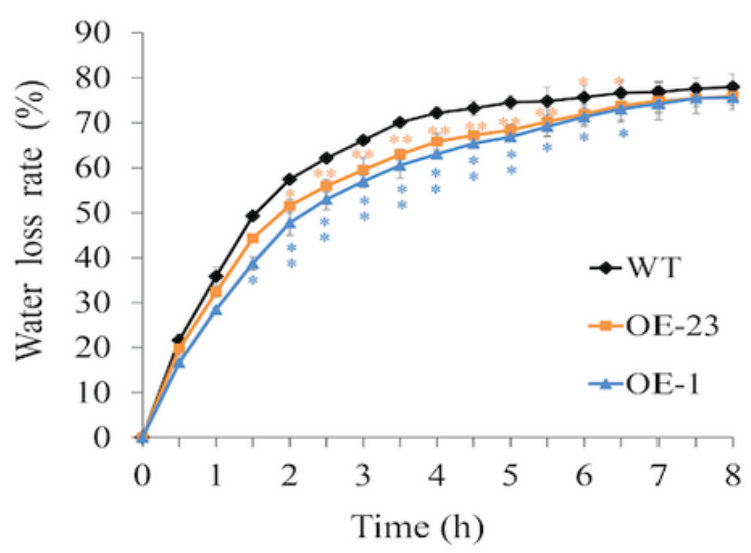

Figure 4

Drought stress analysis of wild-type plants and transgenic plants with OsWRKY97. (A) Performance of 2week-old seedlings from OsWRKY97 transgenic and wild-type plants subjected to drought stress without water for 15 days and then recovered for 3 days. The experiment contained three biological replicates. (B) Survival rates of transgenic and WT plants under drought stress. Values represent the mean $\pm S D(n=3)$ from three independent biological experiments and every replicate contains 12 individual plants.

Statistically significant differences between WT and OE lines were indicated by asterisks (T-test, $* \mathrm{p}<$ $0.05, * * p<0.01$ ). (C) Rate of water loss by detached leaves from control and transgenic plants. It is expressed as percentage of initial fresh weight. Values are the mean $\pm S D(n=3)$ from three independent 
biological experiments and every replicate contains 5 individual plants. The asterisk represents the statistically significant difference at the same time between the WT and OE lines (T-test, $* \mathrm{p}<0.05, * * \mathrm{p}<$ $0.01)$.

A

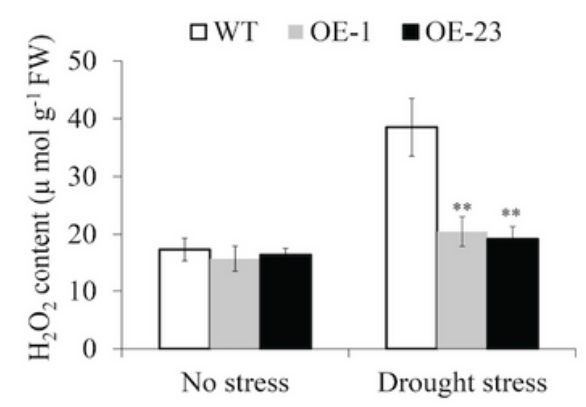

$\mathrm{D}$

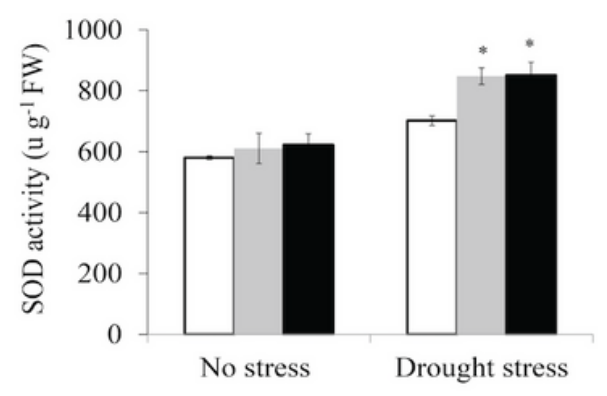

B

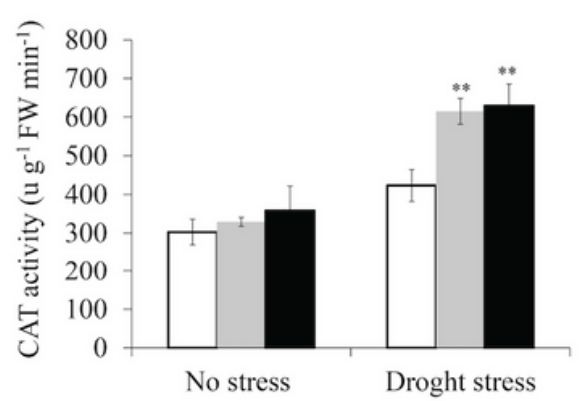

E

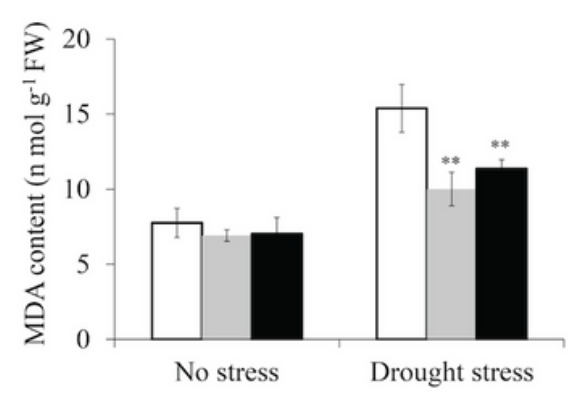

$\mathrm{C}$

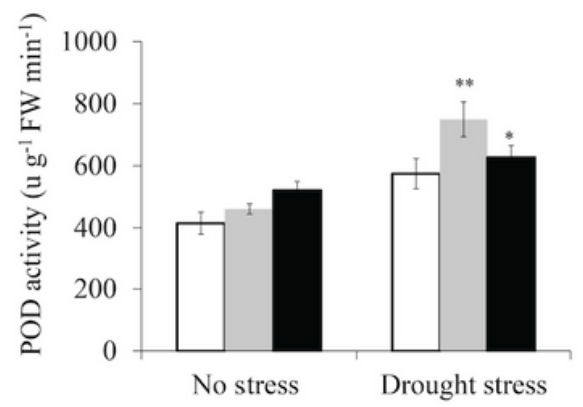

F

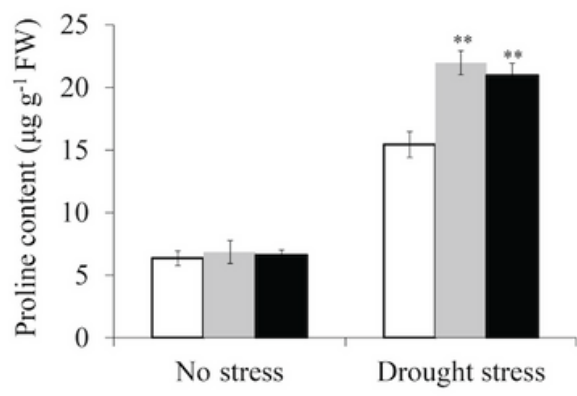

\section{Figure 5}

Physiological parameters of OE plants under Osmotic Stress. Two-week-old rice was transferred to nutrient solution with or without $20 \%$ PEG6000 (A) H2O2 content (B-D) CAT activity, POD activity, SOD activity. (E) MDA content (F) proline content. Values are shown as the mean $\pm S D(n=3)$ and the experiments were performed with at least three biological repetitions. The asterisk represents the statistically significant difference between the WT and OE lines under osmotic stress (T-test, $* p<0.05$, $* * p<0.01)$. 
A

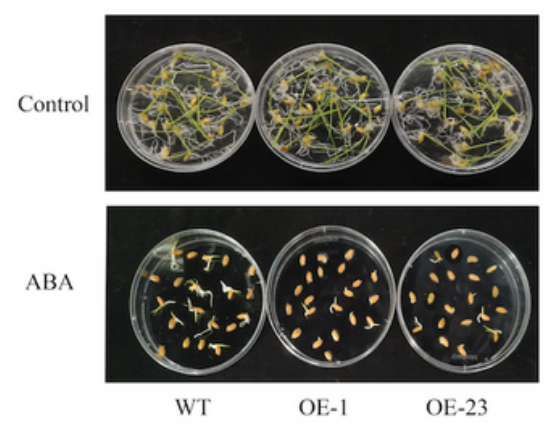

$\mathrm{D}$

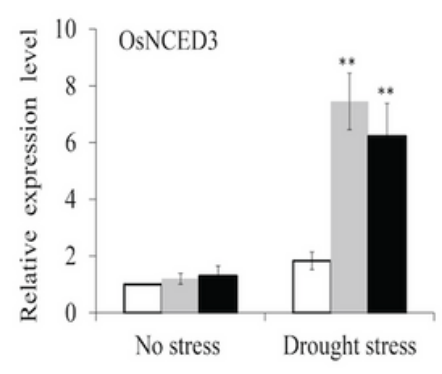

B

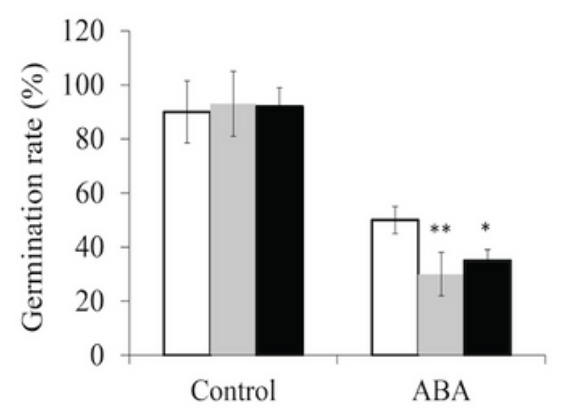

C

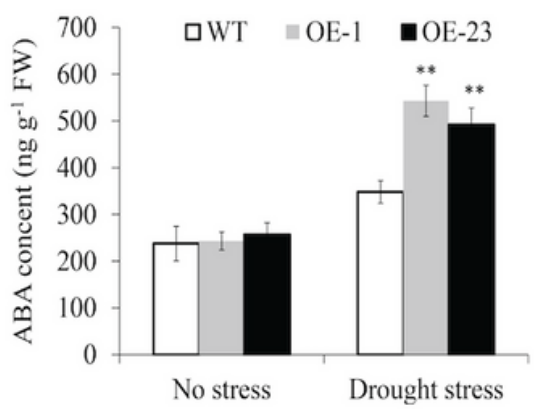

\section{Figure 6}

$A B A$ accumulation and sensitivity of OE plants. The growth performance $(A)$ and The Germination rates (B) of OE and WT seeds under ABA treatment. Values are shown as the mean \pm SD from three biological experiments, with 20 plants in each repeat. The asterisk represents the statistically significant difference between the WT and OE lines under exogenous ABA stress (T-test, $* p<0.05, * * p<0.01$ ). (C) ABA contents of OE and WT plants under normal and drought stress conditions. Results are means \pm SD from three independent biological experiments. (D)Real-time PCR analysis of the expression of ABA biosynthesis and responsive genes under normal and drought stress conditions. The data are represented as the mean $\pm S D$, with three biological experiments. The asterisk represents the statistically significant difference between the WT and OE lines under osmotic stress (T-test, $* \mathrm{p}<0.05, * * \mathrm{p}<0.01$ ).

\section{Supplementary Files}

This is a list of supplementary files associated with this preprint. Click to download.

- Additionalfile2.docx

- Additionalfile3.docx

- Additionalfile1.docx 